\title{
Study on Security Issue in Open Source SIP Server
}

\author{
Muhammad Yeasir Arafat ${ }^{1}$, Muhammad Morshed Alam ${ }^{1} \&$ Feroz Ahmed ${ }^{1}$ \\ 1 Department of Electrical and Electronic Engineering, School of Engineering and Computer Science, \\ Independent University, Bangladesh \\ Correspondence: Muhammad Yeasir Arafat, School of Engineering and Computer Science, Independent \\ University, Bangladesh. Tel: 88-017-19301525. E-mail: yeasir08@yahoo.com
}

Received: January 7, 2014

doi:10.5539/mas.v8n2p124
Accepted: March 6, $2014 \quad$ Online Published: March 11, 2014

URL: http://dx.doi.org/10.5539/mas.v8n2p124

\begin{abstract}
Session Initiation Protocol (SIP) is a core protocol for real-time communication networks, including voice over internet protocol (VoIP). In this paper, author's ensured security for asterisk based SIP server using packet filtering firewall tools know as iptables. Rules are applied at Linux iptables on the basis of respective port numbers, allowing and disallowing particular IP address or IP addresses with subnet. To protect the SIP server from external attack rules are applied at Linux iptables for the useful protocols likes TCP, UDP, RTP and ICMP. A popular simulation software or network protocol analyzer known as Wireshark is used to illustrate how the iptables rules worked that applied for above protocols and shows the changes before and after applying rules. This paper also shows the asterisk server monitoring using the Linux kernel log files and asterisk command line interface (CLI) that shows the successful, unsuccessful SSH login sessions and web based login (HTTPS) with IP addresses list, SIP register request to the SIP server. In this paper, our present approach helps to prevent the SIP server from unauthorized access and attacks.
\end{abstract}

Keywords: VoIP, SSH, SIP, SDP, UDP, TCP, RTP, ICMP

\section{Introduction}

VoIP may be the fastest growing technology that includes routing voice conversations over IP-based network. The flexibleness of the VoIP system convergence of voice and data networks brings with it additional security requirements. SIP servers are vital network elements that endow SIP endpoints to switch messages, register user position, and faultlessly move between networks. SIP servers permit network operators to determine routing and security principles, authenticate clients and control user locations. SIP server applications might take many forms, however the SIP standard defines three general kinds of server functionality that applies to all or any proxy, redirect and registrar servers. SIP server submissions may take numerous forms, but the SIP benchmark defines three general kinds of server functionality that applies to all proxy, redirect and registrar servers.

One of the very most common attacks is denial of service (DoS). After a session has been established with SIP the particular media transfer is transmitted with the true time transport protocol (RTP). In transport layer SIP uses user datagram protocol (UDP). Today it becomes challenge to provide extreme level security for the VoIP server. To ensure security for SIP based asterisk server a system administrator has to follow some steps such as change the default user name (root) and port (22) for remote SSH login to the SIP server, use packet filtering rules for SSH port (22) usually transport control protocol (TCP) packets based on the IP address at Linux iptables, as some open source PBX software provide web based login known as graphical user interface so apply packet filtering rules for HTTPS \& TCP packets with port 443, allow SIP user agents according to IP block by applying rules at iptables for the port 5060 with UDP packets. To prevent internet control message protocol (ICMP) flood attack administrator can allow IP addresses with subnet for ICMP echo ping and trace route request to the SIP sever and block rest of the IP address of the world. Monitoring the Linux kernel log files which usually at /var directory is very important because here administrator can see the all the remote Secure Shell (SSH) login, web based login to the SIP server with the host IP address list. Data log gives the list of registered and unregistered SIP peers with IP addresses and port number which is more important to see the unauthorized attack. Above things will discuss in details bellow with the network protocol analyzer Wireshark. Here Wireshark simulation will show the differences between before and after applying packet filtering rules to the Linux kernel.

Wireshark is a free and open-source packet analyzer. It has a rich and powerful feature set and runs on most 
computing platforms including Windows, OS X, Linux, and UNIX. Network professionals, security experts, developers, and educators around the world use it regularly. Wireshark is very similar to tcpdump, but has a graphical front-end, plus some integrated sorting and filtering. It is used for network troubleshooting, analysis, software and communications protocol development, options. Wireshark allows the user to put network interface controllers that support promiscuous mode into that mode, in order to see all traffic visible on that interface, not just traffic addressed to one of the interface's configured addresses and broadcast/multicast traffic.

In wireshark data can be captured "from the wire" from a live network connection or read from a file of already-captured packets. Live data can be read from a number of types of network, including Ethernet, IEEE 802.11, PPP, and loopback. Captured network data can be browsed via a GUI. Data display can be refined using a display filter. VoIP calls in the captured traffic can be detected. If encoded in a compatible encoding, the media flow can even be played.

\section{Common Attack in SIP Server}

Since there is such a large difference between IPv4 and IPv6, they cannot communicate directly with each other. A system that is capable of handling IPv6 traffic can be made backward compatible, but an already deployed system that handles only IPv4 is not able to handle IPv6 datagram. This means that a major upgrade process would need to take place, involving hundreds of millions of machines, in order to make a complete transition to IPv6. This way is too expensive and time consuming and in any case will not happen overnight. The network world will most likely see a gradual transition to IPv6, where IPv6 will be integrated into the IPv4 world that exists today. There are different kinds of technologies which can be applied such as dual stack, tunnelling, and translation. At present, three transition mechanisms, dual stack, tunneling, and translation, are proposed to solve the problems due to the co-existence of IPv6 and IPv4. Over several transition techniques have been used and tested for the communications between different networks to ensure IPv4 and IPv6 interoperability. Therefore, to make decision on the best suited transition methods, it is really important to have an overview of the current IPv4 networks. In addition, enterprises must analyze needed functionalities, scalability, and securities in the corporation.

\subsection{TCP SYN Flood Attacks}

TCP encompasses a full handshake between sender and receiver before data packets are dispatched. The starting scheme drives a Synchronize (SYN) request in Figure 1. The obtaining system propels an acknowledgement (ACK) having its own SYN request. Then the dispatching system sends back its own ACK and connection can start between the two schemes. If the obtaining system is dispatched a SYNX package but does not obtain an ACKY +1 to the SYNY it sends back to the senders, the receiver will resend a new ACK + SYNY after some time has passed in Figure 1. The processor and memory resources at the obtaining scheme are booked for this TCP SYN demand until a timeout occurs. The TCP SYN attack uses three-way handshake involving the sending system and the receiving system by dispatching huge volumes of TCP SYN packets to the sufferer system with spoofed source IP addresses, so the casualty scheme answers to a non-requesting scheme with the ACK $+\mathrm{SYN}$.

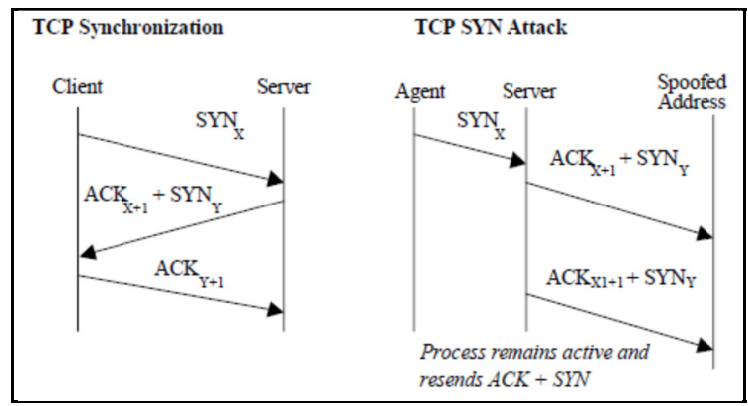

Figure 1. TCP synchronization and TCP SYN attack

When a large volume of SYN requests are being processed by a server and none of the ACK $+\mathrm{SYN}$ responses are returned, the server commences to operate out of processor and memory resources. Ultimately, if the amount of TCP SYN attack requests is huge and they continue over time the victim system will come to an end of resources and that will struggle to answer any legal users. 


\subsection{UDP Flood Attack}

In UDP inundate attack attacker drives large amount of UDP packets to a sufferer system, because of which there is dispersion of the network and the decrease of accessible bandwidth for justifiable service requests to the sufferer system. A UDP inundate attack is possible when an attacker sends a UDP packet to a arbitrary port on the sufferer system. When the sufferer system obtains a UDP packet, it will choose what application is waiting on the destination port. When it realizes there is no application that is waiting on the port, it will assemble an ICMP package of place visited unreachable to the false source address. If ample UDP packets are delivered to ports of the sufferer, the systems will proceed down. UDP inundate attacks might also fill the bandwidth of connections located around the sufferer system that depending on the network architecture and line-speed. This can occasionally cause systems connected to a network near a sufferer system to know-how problems with their connectivity.

\subsection{ICMP Attacks}

Internet Control Message Protocol (ICMP) is an error reporting and analytic utility and it is considered as part of internet Protocol (IP) suite. Whereas this protocol is extremely imperative for ensuring accurate data allocation, it may be exploited by malicious users for conducting different denial of service (DoS) attacks. ICMP inundate attacks, which enables users to drive an echo packet to the host to test check if it's living or not. Expressly throughout a DDoS ICMP inundate attack the agents drive huge volumes of ICMP_ECHO_REPLY packets (ping) to the sufferer. These packets request reply from the sufferer and this consequences in saturation of the bandwidth of the sufferer's network connection. Throughout an ICMP inundate attack the source IP address may be spoofed.

\subsection{Memory Depletion Attacks}

When a server acknowledges a SIP message, it has to shop little chunks of information. The time for which such information portions are retained depends on the server mode - stateful or stateless. While the transaction is being performed the data is kept in memory and it is deleted only one time the transaction is closed or timed out. The most common attack is a TCP SYN inundates strike. The server is inundated with packets with the SYN flag set. The server assigns the essential resources and answers with packets with SYN+ACK flags. The attacker stays on sending new packets with SYN banners, and does not reply to packets dispatched by the server. The server rapidly depletes all memory resources for a new TCP connection and starts denying normal demands. Another case of this kind of attack would be to drive exceedingly fragmented packets with certain parts intentionally misplaced. The server endeavors to demand the missing parts and stores the obtained packets in memory. The futile information is then retained on the server until it is timed out.

\subsection{CPU Depletion}

An added way to competently limit the server's capability to method regular demands is central processing unit (CPU) depletion. A higher load might be the result of a higher amount of requests received or by receiving requests requiring extra complex calculations. The server can become inundated with ICMP packets but sending malformed REGISTER messages creates the similar effect with a considerably smaller amount of messages. This really is due to the undeniable fact that messages are studied following the server receives them. Even though the server has the capacity to process hundreds of regular messages, it may be simply forced to do different calculations using malformed messages with fake or invalid data or sent from nonexistent user accounts. Absurdly, enabled authentication on a server can punctual off more demanding procedures, which makes it simpler to decrease CPU resources. When a server uses certificate, the attacker may drive a note by having an invalid certificate. In the end, the server works out that this certificate is invalid nevertheless the processing of the message had already consumed much of server resources. The prospective is flooded with more messages than it may process at a given time. As SIP is a text-based protocol; it has to parse each incoming message. If SIP authentication info is supplied in the inundating message, it has to assess if the user is authorized to get access to the service (assimilate authentication). A specific case is once the target CPU will not extend its operation because it is awaiting input from other entities, such as for instance a database or the domain name system (DNS) service.

\subsection{Bandwidth Depletion Attacks}

This sort of attack does not consume resources of the physical server but alternatively the capability of the hyperlink connecting the server to the network. When the hyperlink is unable to transmit regular packets, they're unnecessary before they are able to arrive at the SIP server. For this reason it is not possible to differentiate between regular and malicious packets. Utilizing the UDP protocol to transmit SIP messages makes the 
condition even worst. For reasons, attackers utilize the stateless UDP protocol with the utmost packet size.

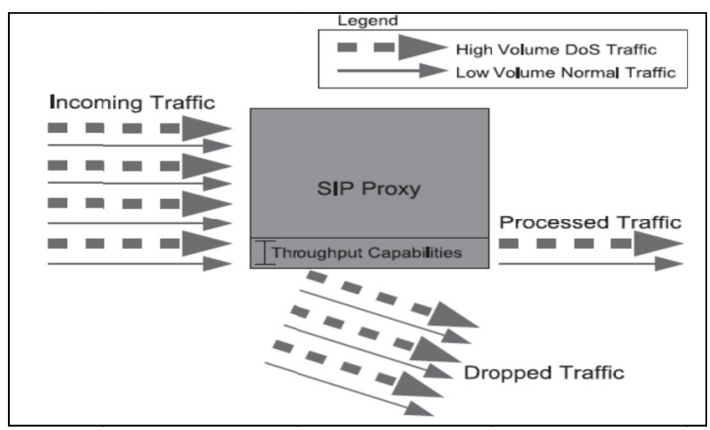

Figure 2. Overview of a DoS flooding attack

Because of the server's limited processing capabilities a allotment of normal demands cannot be processed in case a high load of malicious messages are targeted towards the server.

\section{Proposed Solutions using Linux Operating System and IP tables}

Linux is open-source operating systems, since it has the key advantageous features where users can modify the code. It is made in this method that it can operate on different types of hardware. The Linux kernel is issued under the general public license version 2 (GPLv2). IP firewalls support different types of filters. IP firewalls be adept of do filters and firewall policy by usernames, by group IDs and user profiles, by source and destination ports, by source host and destination hosts, by uniform resource locator (URL), by IP addresses, by packet ID flags, by protocols and also including filtering by media access control (MAC) address.

\subsection{IP Tables}

IPtables offers moderately higher speed and reliability compared to the other firewall tools. Being fully a Linux product, its integration with the functioning operating system (OS) is also more powerful and reliable. It keeps a stateful route of each connection passing through it and tries to anticipate feature actions. Its capability of filtering packets on the basis of MAC address makes it a appalling security system. It may filter out attacks using malformed packets and can restrict access from locally attached servers to other networks regardless of their valid IP addresses. Network address translation (NAT) with masquerading convenience of IPtables helps it to conceal internal network IP sub networks behind one or perhaps a small poll of external IP addresses. NAT and masquerading enables the firewall system to open and close ports on the gateway. The rate-limiting characteristic of IPtables can block attacks even from some forms of DoS attacks.

\section{(i) Firewall Rules}

Different firewalls usually supply distinct direct reasoning with distinct parameters. But some absolutely vital components are universal to all. Each of them permits an action to be distinct allowing or banning specific network traffic. Also, those all allow checking for most critical elements in packets like IP addresses, ports and protocol. IP Tables is a command line tool for writing and executing of Firewall rules. One of the very important functionalities of IP Tables firewall is stateful inspection (S.I), which routinely opens only the ports necessary for internal packets to access the Internet. It only allows transfer of packets which are defined in firewall rules and which are section of established connections.

(ii) Firewall Chains

IP Tables group policy in chains. Different network packets are route by different chains:

- Incoming traffic - packets for firewall (INPUT chain).

- Forwarding traffic - incoming packets for another appliance (FORWARD chain).

- Outgoing traffic - packets generated by firewall (OUTPUT chain).

Forms of tables have been in IP tables are shown below:

- Filter table- This table principle is used for packet filtering in IP network.

- NAT table- This table policy is not used for packet filtering however it slightly offers NAT/PAT capabilities and IP masquerading. 
- Mangle table- This table is employed to change packet fields and is also used to mark packets for later filtering.

The available targets or actions for policy in filter table chains are-

- $\quad$ ACCEPT- This target is requested those packets that are reliable and can be accepted through the firewall.

- $\quad$ REJECT- This target rules is requested those packets that are to be fallen but an ICMP reply will be provided for the inventor for information on what occurring to the packet.

- DROP- This target is placed on those packets that are to be just fallen without dispatching any ICMP reply like it never reached firewall.

(iii) Firewall Rules Parameters

Each rule recognizes explicit kind of network traffic. In alignment to permit this identification parameter for identification of specific network packets must be set for each direct.

Different types of parameters are:

- IP addresses - It can be destination or source IP address also, is often as just one IP, network IP block or IP range.

- $\quad$ Ports - It can be destination or source port also, is often as just one port, port range or port array.

- $\quad$ Protocol - It can be submitted to TCP, UDP, and ICMP or all together.

- Interface - It can be incoming or outgoing interface.

- TTL (Time to Live) field residing in the IP headers.

- ToS (Type of Service) field residing in the IP headers.

- Length of packet.

- MAC source address.

- Syn flag - identification of new connection.

- ICMP type.

\section{Result and Analysis}

\subsection{Basic Security for the Linux Server}

Apply rules for remote Secure Shell (SSH) login to the Linux kernel such as changing the default username, password and port number. Do not use the default username root and port 22 as the SSH port. To ensure the primary level security, a system administrator should change these default user name and port number of SSH form the Linux kernel just following the bellow steps:

Add a New Username and Password: To add and give a strong password for a new user has to give the command:

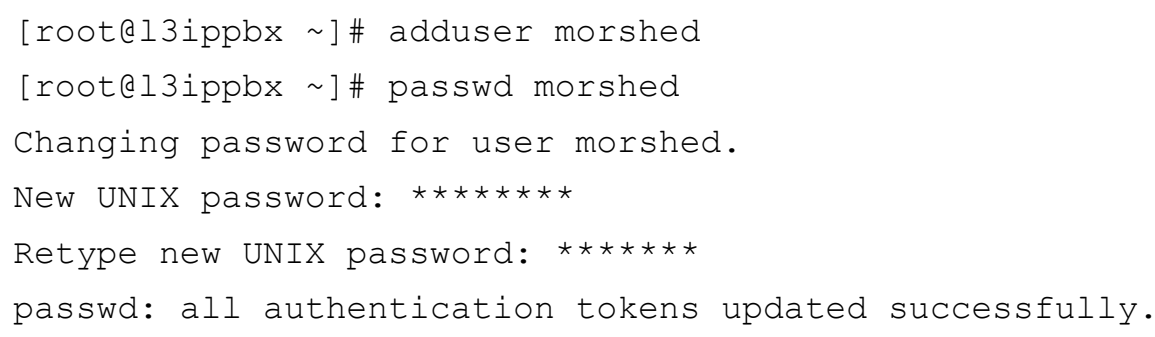

Now to ensure more security for a sever change the default SSH port 22. To do this administrator has to change the directory of the linux as given bellow:

roota13ippbx ssh]\# cd /etc/ssh

"etc" is a essential directory of linux kernel which provides the host specific system configuration files and has to move to the SSH directory. Here administrator looking for a file which name is sshd_config, inside this file administrator can add the login permission of the new user here it is "morshed" and also can change the SSH port 10 instead of default port 22 by using the following command-

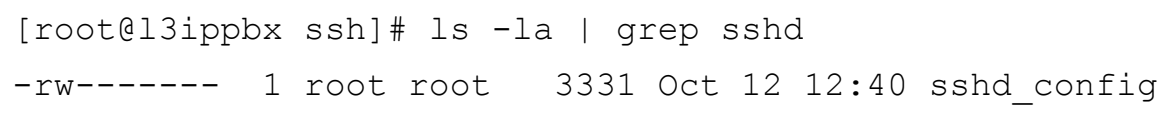


[rootel3ippbx ssh]\# vi sshd_config

\$OpenBSD: sshd_config,v 1.73 2005/12/06 22:38:28 reyk Exp \$

allowUsers morshed

port 10

[rootal3ippbx ssh]\#/etc/init.d/sshd restart

Stopping sshd: [OK]

Starting sshd: [OK]

Now SSH remote login default username root is disabled and Linux kernel allow only user "morshed" which is created earlier for the remote SSH login and port 10 will be used instead of 22 with the hostname or IP address of the asterisk server. Then the user "morshed" can login to the "root" via bellow command with root password:

[morshedel3ippbx ]\$ su -

Password:

[rootal3ippbx ] \#

So this may help something administrator to provide multilevel password protection to his server and unauthorized remote sessions to his server from different hosts. To monitor the authorized sessions, administrator can check the $\log$ file of the Linux and asterisk regularly. From the log file we could see hundreds of failed register attempts from the several ip addresses in internet. This log may supply vital information to fight hackers because it shows the scale of the issue, the accounts they are attempting to crack, nature of attack and also attacker IP address.

\subsection{Log Monitoring}

The useful variable directory "var" which contains the entire log file of Linux kernel and also asterisk by using following command:

[rootal3ippbx ]\# tail -f /var/log/secure

Oct 12 10:34:24 localhost sshd[24809]: Failed password for root from 203.76.99.103 port 49908 ssh2

oct 12 10:34:28 localhost sshd[24809]: Accepted password for root from 203.76.99.103 port 49908 ssh2

oct 12 10:34:28 localhost sshd[24809]: pam_unix(sshd:session): session opened for user root by (uid=0)

Oct 12 10:34:37 localhost sudo: root : TTY=unknown; PWD=/root ; USER=root ; COMMAND=/usr/sbin/asterisk -vVVVVrx core show channels

oct 12 13:31:21 localhost sshd[27343]: Disconnecting: Too many authentication failures for root

Oct 12 13:31:21 localhost sshd[27340]: PAM 3 more authentication failures; logname $=u i d=0$ euid=0 tty=ssh ruser= rhost=67.198.172.202 user=root

Oct 12 13:31:21 localhost sshd[27340]: PAM service(sshd) ignoring max retries; $4>3$

Oct 12 13:31:23 localhost sshd[27348] : reverse mapping checking getaddrinfo for 67.198.172.202. customer.krypt.com failed - POSSIBLE BREAK-IN ATTEMPT!

Oct 12 13:31:23 localhost sshd[27348]: pam_unix(sshd:auth): authentication failure; logname $=$ uid=0 euid=0 tty=ssh ruser $\bar{r}=$ rhost $=67.198 .172 .202$ user $=$ root Oct 12 13:31:25 localhost sshd[27348]: Failed password for root from 67.198 .172 .202 port 3778 ssh2

Oct 12 13:31:32 localhost last message repeated 3 times

oct 12 10:34:47 localhost last message repeated 9 times

[rootel3ippbx ]\#tail -f/var/log/elastix/audit.log

[Oct 10 19:58:36] NAVIGATION admin: User admin visited "PBX >> PBX Configuration" from 203.76.99.103. 
[Oct 10 20:00:03] NAVIGATION admin: User admin visited "PBX >> Tools >> Asterisk-Cli" from 203.76.99.103.

[Oct 10 20:00:09] NAVIGATION admin: User admin visited "PBX >> Tools >> Asterisk-Cli" from 203.76.99.103.

Directories to see the Linux kernel and full asterisk logs:

[rootel3ippbx ] \#tail -f / var/log/messages

[rootal3ippbx ] \#tail -f /var/log/asterisk/full

The Linux kernel log also gives the all successful remote SSH login session with IP address or hostname, login time-date-month, username and time of the active sessions. To view this administrator can run a single command that given bellow Figure 3 .

\begin{tabular}{|c|c|c|c|c|c|c|c|c|}
\hline \multicolumn{9}{|c|}{ [root@13ippbx ]\# last } \\
\hline iptsp & pts/1 & 203.76 .99 .103 & Sat & Nov & 2 & $14: 09$ & $-14: 20$ & $(00: 11)$ \\
\hline iptsp & pts/1 & 203.76 .99 .103 & Sat & Nov & 2 & $11: 35$ & $-11: 38$ & $(00: 02)$ \\
\hline iptsp & pts/1 & 203.76 .113 .226 & Fri & Nov & 1 & $00: 47$ & $-00: 51$ & $(00: 04)$ \\
\hline iptsp & pts/1 & 203.76 .113 .226 & Thu & Oct & 31 & $23: 24$ & $-23: 27$ & $(00: 03)$ \\
\hline iptsp & pts/1 & 203.76 .99 .103 & Thu & Oct & 31 & $14: 18$ & $-14: 20$ & $(00: 02)$ \\
\hline iptsp & pts/1 & 203.76 .99 .103 & Thu & Oct & 31 & $12: 10$ & $-12: 51$ & $(00: 41)$ \\
\hline
\end{tabular}

Figure 3. Host IP addresses list of SSH login to the SIP server

Some useful Asterisk CLI commands help the administrators to see registered extensions with corresponding IP addresses, the active number of calls and channels through bellow Figures 4-6 commands

[root@13ippx $\sim \#$ asterisk $-r$

$13 i \mathrm{ppx} * \mathrm{CLI}>$

\begin{tabular}{|c|c|c|c|c|c|c|}
\hline \multicolumn{7}{|c|}{13 ippbx*CLI> sip show peers } \\
\hline Name/username & Host & Dyn & For & ACL & Port & Status \\
\hline $1000 / 1000$ & 10.111 .10 .122 & D & $\mathrm{N}$ & $\mathrm{A}$ & 5062 & OK (55 ms) \\
\hline 1001 & (Unspecified) & D & $\mathrm{N}$ & $\mathrm{A}$ & 0 & ONKWNONN \\
\hline $1002 / 1002$ & 123.200 .9 .2 & $\mathrm{D}$ & $\mathrm{N}$ & $\mathrm{A}$ & 5062 & OK (54 ms) \\
\hline $1100 / 1100$ & 203.76 .114 .122 & D & $\mathrm{N}$ & $\mathrm{A}$ & 1024 & OK (59 ms) \\
\hline $1212 / 1212$ & 203.76 .99 .234 & D & $N$ & $A$ & 1027 & OK (5 ms) \\
\hline $1222 / 1222$ & 203.76 .99 .234 & D & $\mathrm{N}$ & $\mathrm{A}$ & 5060 & OK (121 ms) \\
\hline $1233 / 1233$ & 203.76 .99 .234 & D & $\mathbb{N}$ & A & 1253 & OK (6 ms) \\
\hline $1616 / 1616$ & 10.111 .13 .124 & $D$ & $\mathrm{~N}$ & $\mathrm{~A}$ & 5060 & OK (4 ms) \\
\hline
\end{tabular}

Figure 4. Registered SIP IP addresses list

To see the active calls or channels just run the bellow command at asterisk CLI showed in Figure 5.

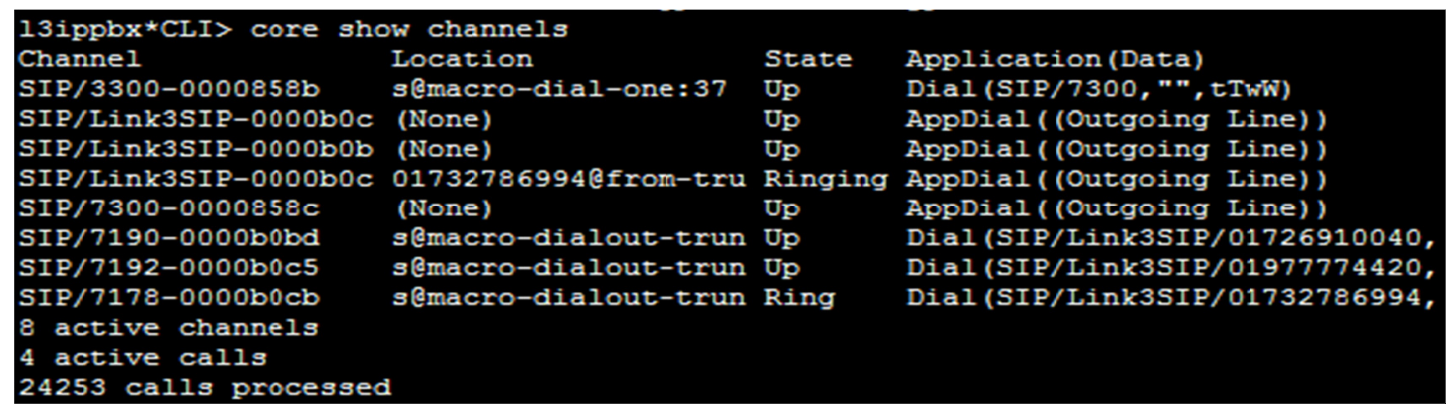

Figure 5. Current active calls list at asterisk CLI 
Here administrator can hang-up above active call of the extension SIP/7190-0000b0bd by using command form asterisk CLI as given bellow:

\section{3ippx*CLI> channel request hangup SIP/7190-0000b0bd}

Command to see the active peers with send and receive packet jitter and also the time duration of calls at asterisk CLI

\begin{tabular}{|c|c|c|c|c|c|c|c|}
\hline \multicolumn{8}{|c|}{ 13ippbx*CLI> sip show channelstats } \\
\hline Peer & Call ID & Duration Recv: Pack & Lost & ई) & Pack & Lost & Jitte: \\
\hline 203.76 .96 .85 & 2faf $635574 f$ & $00: 01: 28 \quad 0000004252$ & 0000000000 & $0.00 \$)$ & 0.00000000004423 & 0000000000 & 0.00 \\
\hline .85 & $182 \mathrm{bad} 216 \mathrm{ee}$ & $: 00: 45 \quad 0000002049$ & 100000000 & $.00 \xi)$ & $0.0000 \quad 0000002263$ & 0000000000 & 00 \\
\hline 34 & $1 \mathrm{e} 4 \mathrm{~d}$ & 540000002448 & 100000000 & $00 \xi)$ & 0.0000000 & 00000 & .000 \\
\hline & $3 a 7$ & 900 & 0000 & $00 \xi)$ & 0.000000 & 0000 & .000 \\
\hline & lead? & 700 & 0000000000 & 0ई) & 0.0000000 & 0000000000 & .000 \\
\hline & $09 f 19$ & 00 & 0000 & อ६) & 0.000000 & 0000 & .000 \\
\hline 34 & 387 & 00: & 0000 & §) & 0.000000 & 0000 & 0.000 \\
\hline & $7 \mathrm{fd}$ & 00 & 000 & ई) & 0000 & 000 & 0.00 \\
\hline & $008 \mathrm{~b} ?$ & 28000 & 0000 & $0.00 \xi)$ & 0.0000000 & 0000 & 0.000 \\
\hline 0.11 & a20d803838c & $00: 00: 370000001130$ & 0000000000 & $0.00 \xi)$ & 0.00000000001133 & $0000000000(0.00 \xi)$ & 0.00 \\
\hline
\end{tabular}

Figure 6. Active peers with send and receive jitter and also the time duration of calls at asterisk CLI

\subsection{Apply Extreme Level Security for SIP Server by Linux IP Tables}

IPtables is firewall systems in Linux kernel which provides the users to change and construct an individual freedom. Net filter is the fundamental concept of IPtables. Actually it's a supply filtering firewall of Linux machine that filters packets by the fields in IP, TCP, UDP, and ICMP packet headers etc. By Applying rules at Linux iptables administrator can allow or disallow a certain IP address or range of IP addresses with on the foundation of port number for a certain asterisk server. To make certain more security for the asterisk server administrator can apply rules at Linux IPtables for remote SSH login, Web Browsing (https, http). SIP is a software layer protocol in TCP IP which can be widely using for VOIP to ascertain a period utilizing the default port 5060 which uses UDP and RTP for voice/data transmission by allowing a certain IP or IP addresses range. Network mapping "nmap" in Linux is a critical command to see the open protocols with port number as bellow command output:

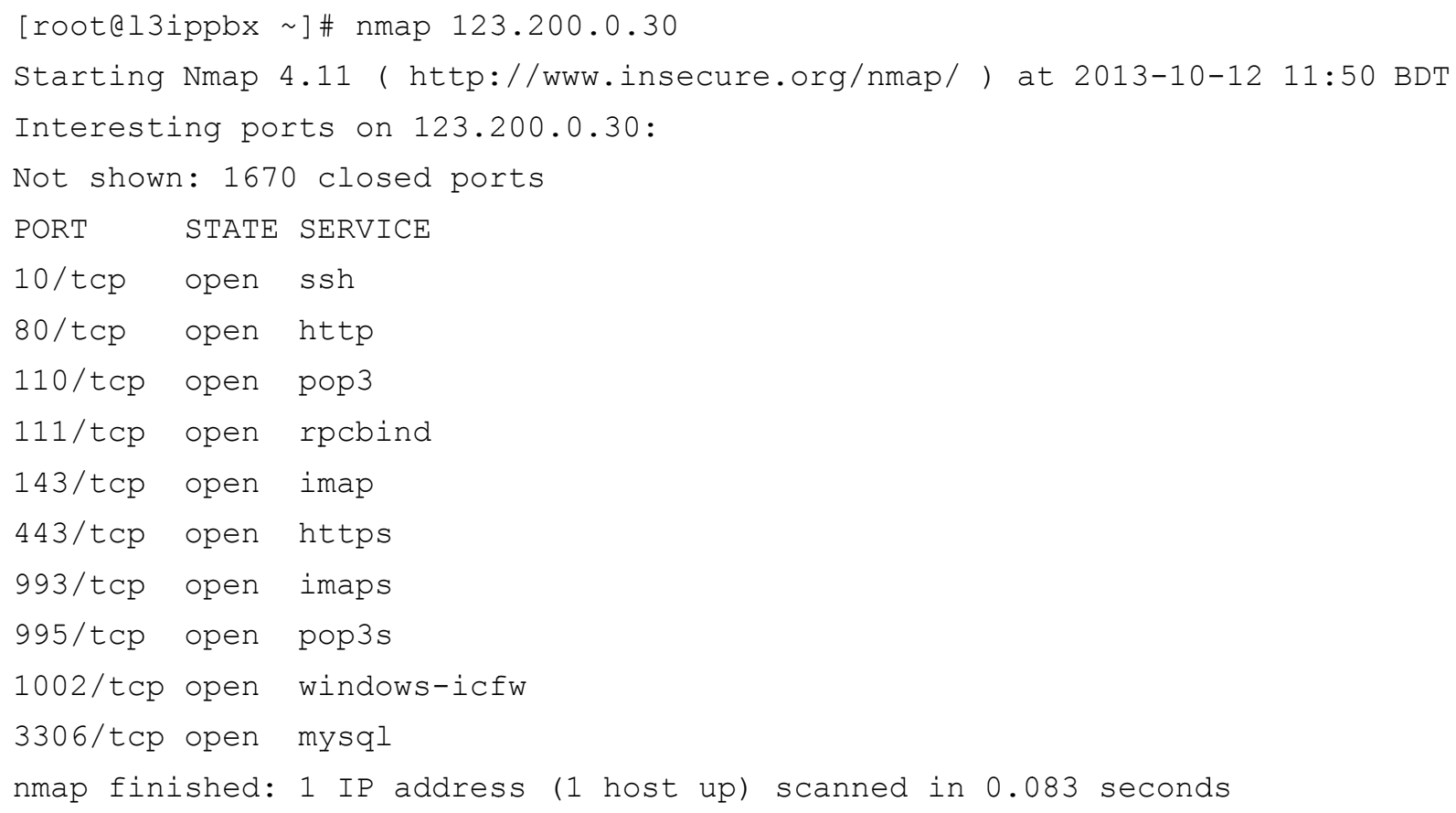

Iptables rules for remote SSH login to the asterisk server:

[rootel3ippx ] \# /sbin/iptables -A INPUT -p tcp -s 203.76.0.0/16--dport 10 -j ACCEPT 


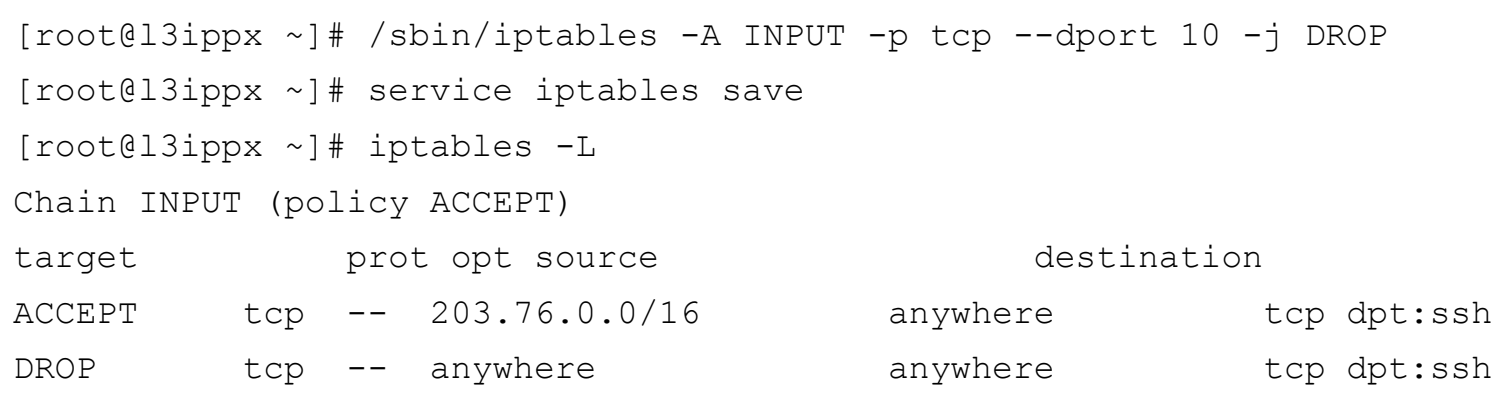

IPtables rules for allowing and disallowing IP addresses for Session Initiation Protocol (SIP) Register request through the default port 5060. Session Initiation Protocol (SIP), an application-layer control (signaling) protocol for creating, modifying, and terminating sessions with a number of applicants. This session integrated internet telephone calls, multimedia conferences, instant messaging, voice, video, interactive games, and virtual reality etc. A SIP message includes a header by having an elective body. These messages are either requests or responses. SIP entity received request and it performs the corresponding action and sends back an answer to the originator of the request. Responses are three digit status codes.

Table 1. SIP Requests

\begin{tabular}{ll}
\hline SIP Request & Response \\
\hline INVITE & Initiates a call by inviting user to participate in session \\
ACK & Confirms that the client has received a final response to an INVITE request \\
BYE & Indicates termination of the call \\
OPTIONS & Used to query the capabilities of a server \\
CANCEL & Cancels a pending request \\
REGISTER & Registers the user agent \\
\hline
\end{tabular}

Table 2. SIP Response Codes

\begin{tabular}{ll}
\hline SIP Response codes & Meanings \\
\hline $1 \mathrm{XX}$ & Informational Responses \\
$2 \mathrm{XX}$ & Successful Responses \\
$3 \mathrm{XX}$ & Redirection Responses \\
$4 \mathrm{XX}$ & Request Failure Responses \\
$5 \mathrm{XX}$ & Server Failure Responses \\
$6 \mathrm{XX}$ & Global Failures Responses \\
\hline
\end{tabular}

Without applying the rules at Linux iptables for SIP default port 5060, it is seen that a user agent with IP address 203.76.99.103 provides 2 register packet requests to the SIP server 123.200.0.36 according to the Figures 4 and 5 at wireshark then the SIP server 123.200.0.36 provides the OPTIONS and successful response code 200 OK as an acknowledgement message to the user agent 203.76.99.103 as given as bellow Figures 7, 8 at wireshark. 


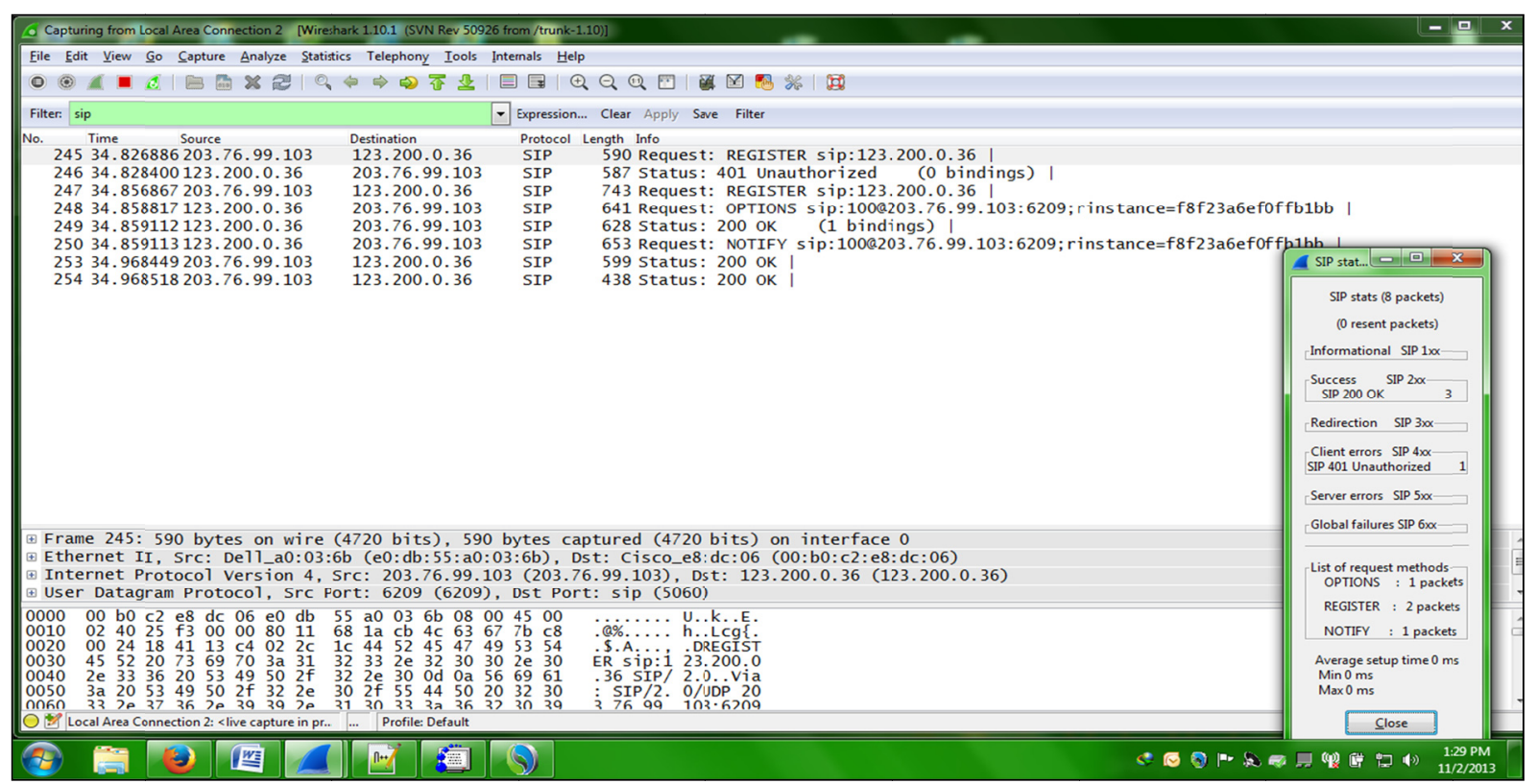

Figure 7. SIP register request packets flow form IP 203.76.99.103 to SIP server 123.200.0.36

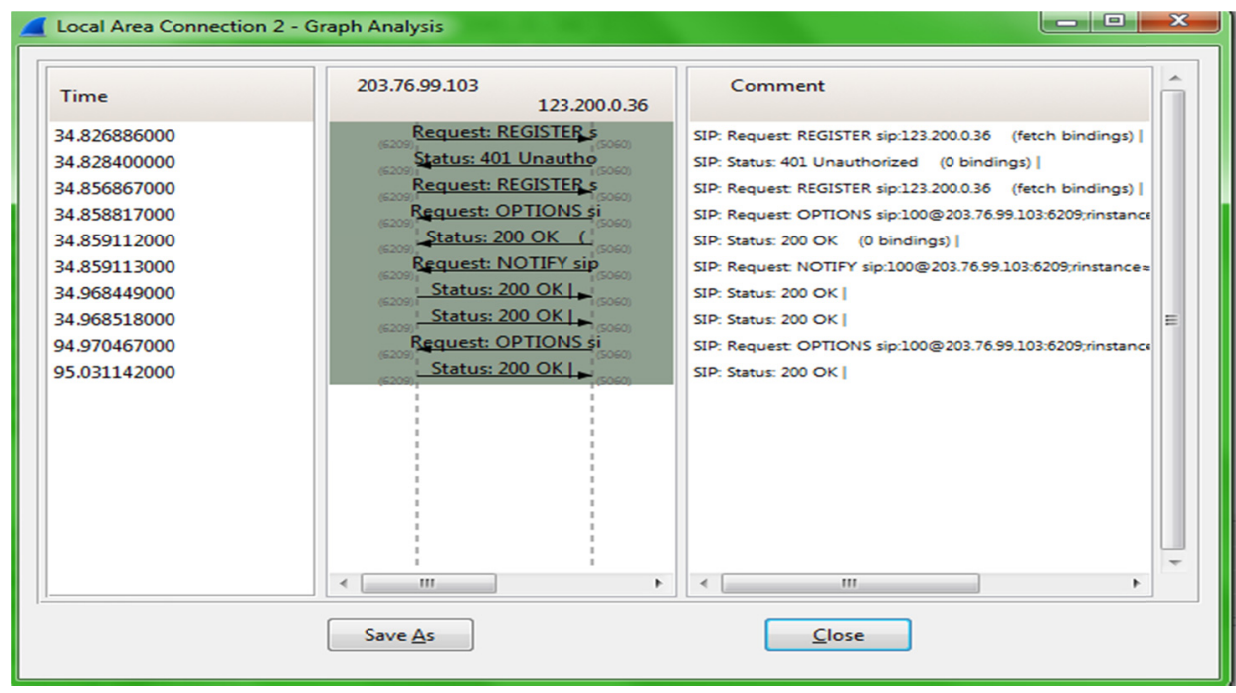

Figure 8. Flow graph of SIP register request form the host 203.76.99.103 to SIP server 123.200.0.36

Now apply the rules at Linux kernel iptables to allow the IP block 203.76.0.0./16 and disallow rest of the IP addresses of the world for SIP Register request through the default port 5060 for the SIP server with IP address 123.200.0.36 by using bellow commands.

[root@13ippx ] \#/sbin/iptables -A INPUT -p udp -s 203.76.0.0/16 --dport 5060 $-j$ ACCEPT

[root@13ippx ]\# /sbin/iptables -A INPUT -p udp --dport 5060 -j DROP

[root@l3ippx ]\# service iptables save

Saving firewall rules to/etc/sysconfig/iptables: [ OK ]

[root@l3ippx ] \# iptables -L

Chain INPUT (policy ACCEPT)

target port opt source destination 


\begin{tabular}{|c|c|c|c|c|}
\hline ACCEPT & udp -- & $203.76 .0 .0 / 16$ & anywhere & udp dpt:sip \\
\hline$R \cap P$ & udp & anywhere & anywhere & udp dpt:sip \\
\hline
\end{tabular}

Now send register request form the user agent with IP address 10.111.11.232 which is not at allowed ip block therefore Linux kernel dropped the SIP register request though the agent 10.111.11.232 send 11 packets of register request to the SIP server 123.200.0.36 according to the wireshark flow graph in Figure 9.

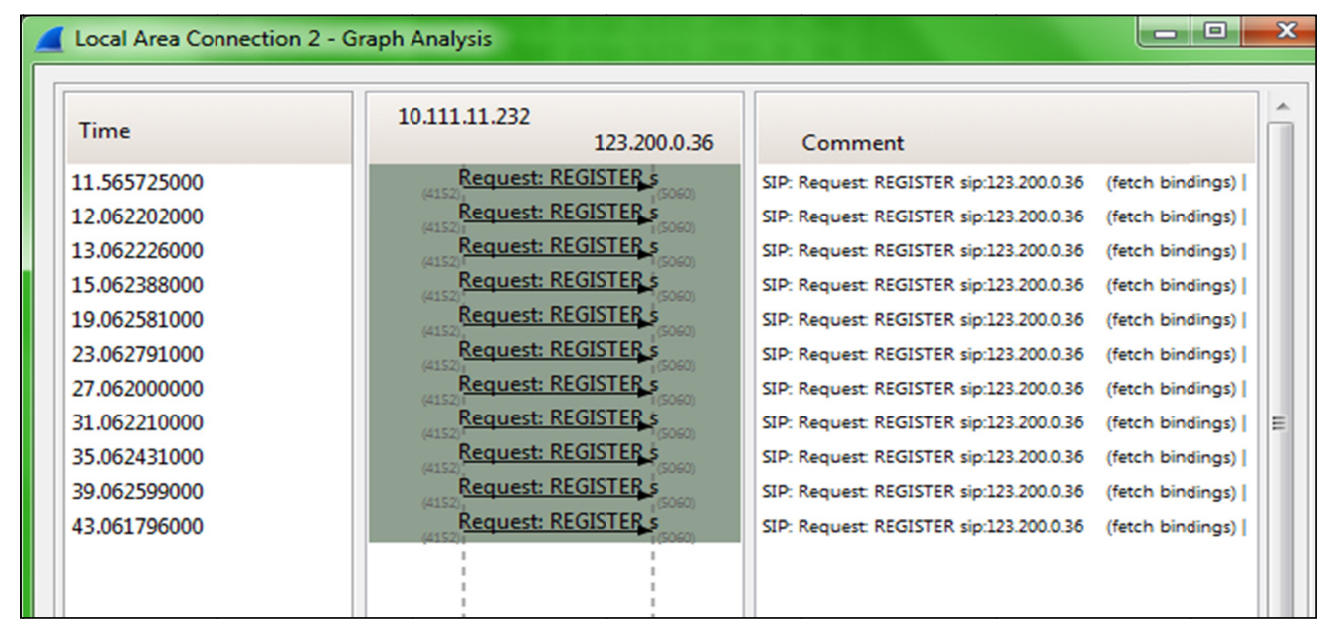

Figure 9. Wireshark flow graph for SIP register request from user agent 10.111.11.232 to SIP server 123.200.36

\subsection{Prevent Web Based Attacks}

As some asterisk based PBX software provides the graphical user interface, so administrator can allow a particular IP or IP address ranges for graphical usually admin login to the server by allowing or blocking the HTTPS protocol port number as given bellow:

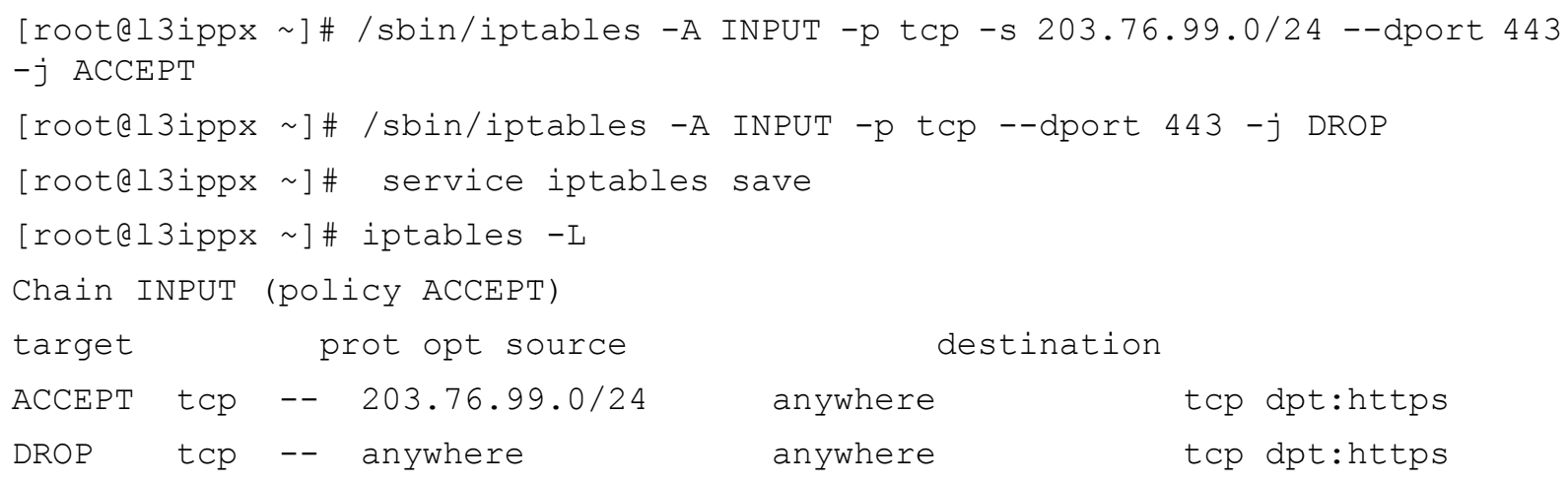

\subsection{Prevent Internet Control Message Protocol (ICMP) Flood Attack}

To confuse the hacker or unauthorized users administrator can allow ICMP ping, trace route request for particular IP address or IP ranges and disallow rest of the IP address of the world for a particular SIP server. 


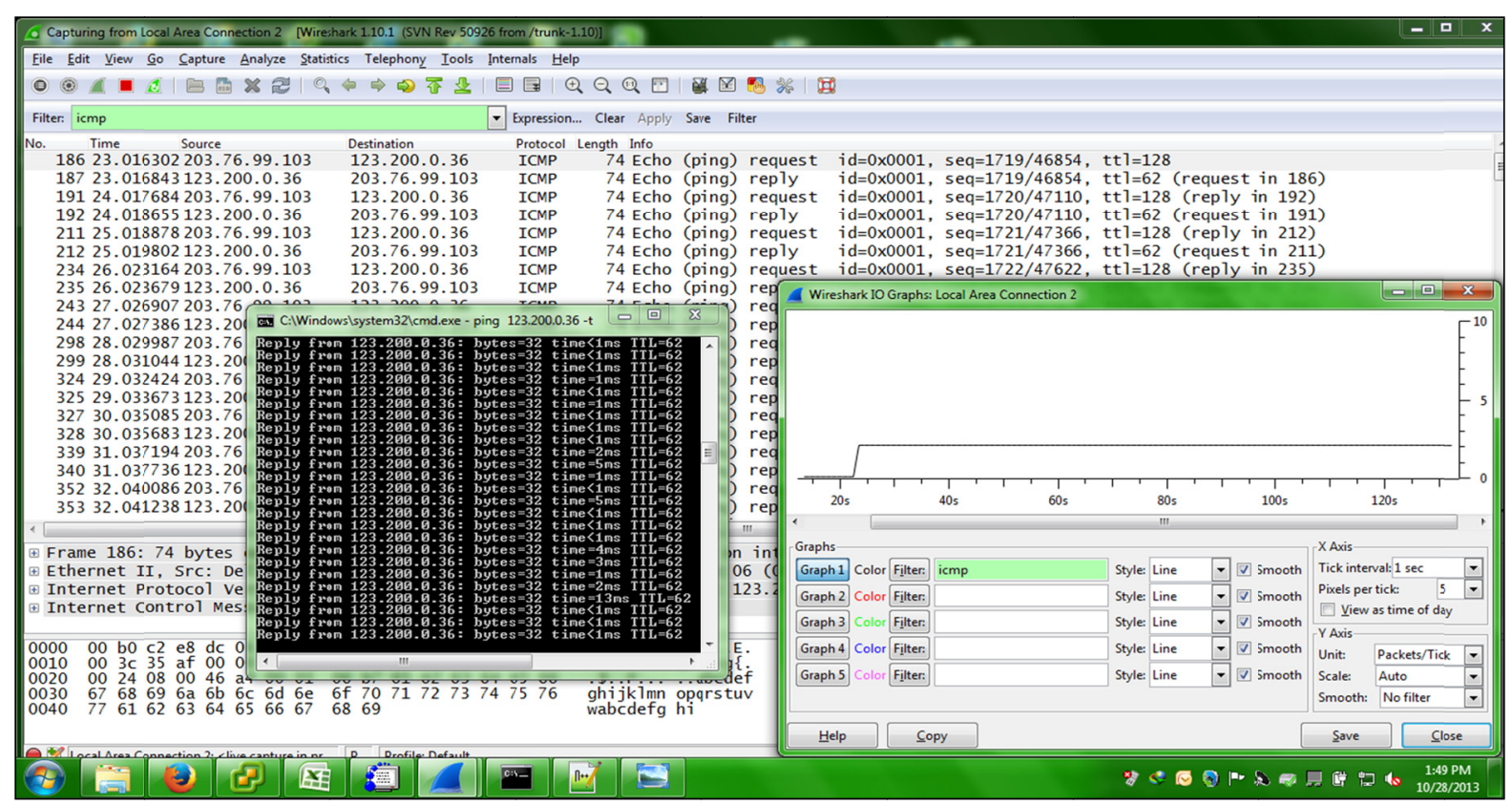

Figure 10. Wireshark view for ping request form 203.76.99.103 to 123.200.0.36 and packet/tick graph at wireshark

ICMP works at the network layer and ICMP flood attack which enables users to send an echo packet to a remote host to check whether it's alive or not. Without applying rules at Linux iptables for ICMP ping request at the asterisk server, it allows input ping request packet from different host and then transmit a echo or reply packet to that host as an acknowledgement as like as Figure 8. Here a host 203.76.99.103 which OS is windows-7 sends ping request to the asterisk server which OS is Red Hat Linux (centos-6.4) with ip address 123.200.0.36. Since still Linux sever do not apply iptables rules for ICMP, host 203.76.99.103 gets continuous TTL response as wireshark shows the ping request from 203.76.99.103 and reply request from 123.200.0.36. Therefore Packet/Tick graph at wireshark gives the continuous line as like as bellow Figure 11.

\begin{tabular}{|c|c|c|c|}
\hline \multicolumn{2}{|c|}{$\triangle$ Local Area Connection 2 - Graph Analysis } & \multicolumn{2}{|c|}{\begin{tabular}{|l|l|l}
0 & 回 & $\mathbf{X}$ \\
\end{tabular}} \\
\hline Time & 203.76.99.103 & Comment & $\hat{\square}$ \\
\hline 17.384633000 & Echo (ping) request & ICMP. Echo (ping) request id $=0 \times 0001$ seq $=2048 / 8, \pi l=128$ & \\
\hline 17.385573000 & Echo (ping) reply & ICMP: Echo (ping) reply id $=0 \times 0001, \mathrm{seq}=2048 / 8, \pi t=62$ (re & $\equiv$ \\
\hline 18.386164000 & Echo (ping) request & ICMP. Echo (ping) request id $=0 \times 0001$ seq $=2049 / 264, t \mathrm{t}=1 \mathrm{i}$ & \\
\hline 18.387542000 & Echo (ping) reply & ICMP: Echo (ping) reply id =0x0001, seq $=2049 / 264, t \mathrm{tl}=62$ & \\
\hline 19.392140000 & Echo (ping) request & ICMP: Echo (ping) request id $=0 \times 0001$ seq $=2050 / 520, t \mathrm{tl}=1 \mathrm{i}$ & \\
\hline 19.392646000 & Echo (ping) reply & ICMP. Echo (ping) reply id $=0 \times 0001, \mathrm{seq}=2050 / 520, t \mathrm{tl}=62$ & \\
\hline 20.393347000 & Echo (ping) request & ICMP: Echo (ping) request id $=0 \times 0001$ seq $=2051 / 776, t \mathrm{tl}=1 \mathrm{i}$ & \\
\hline 20.393827000 & Echo (ping) reply & ICMP: Echo (ping) reply id $=0 \times 0001$, seq $=2051 / 776, t$ tl $=62$ & \\
\hline
\end{tabular}

Figure 11. Wireshark flow graph for ping request form 203.76.99.103 to 123.200.0.36

Without ICMP rules at Linux firewall the trace route command by host 203.76.99.103 gives the complete path to reach the SIP server 123.200.0.36 as given bellow Figure 12 . 


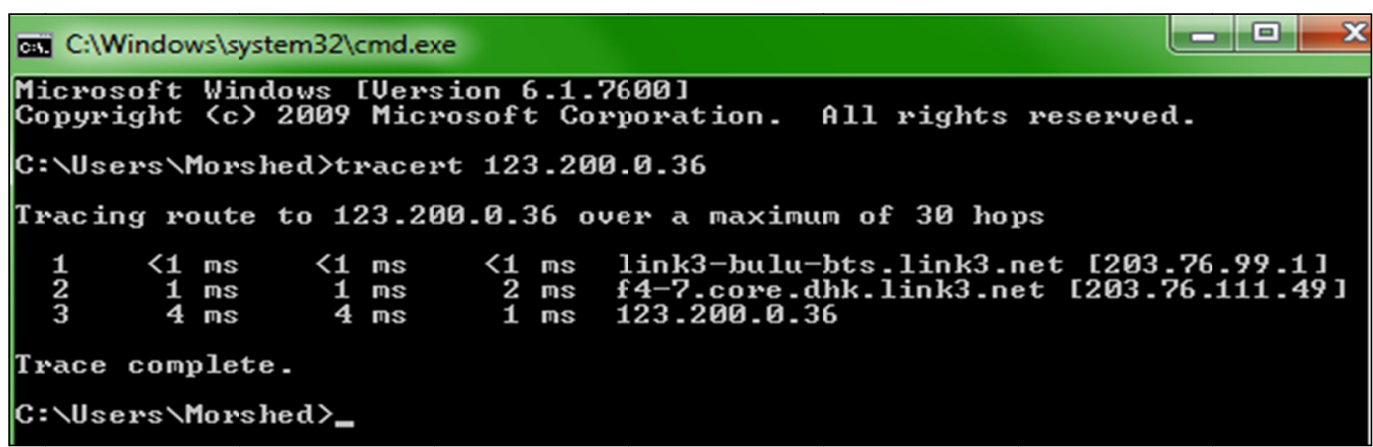

Figure 12. Trace route path from the host 203.76.99.103 to SIP server 123.200.0.36

Now apply the rules for ICMP at iptables of Linux kernel by using bellow commands, here input icmp echo-request of the server with IP 123.200.0.36 is allowed only for IP block 203.76.99.0/24 and rest of the IP addresses of the world will be dropped when they send the icmp ping request to this asterisk server with IP address 123.200.0.36 by the Linux kernel.
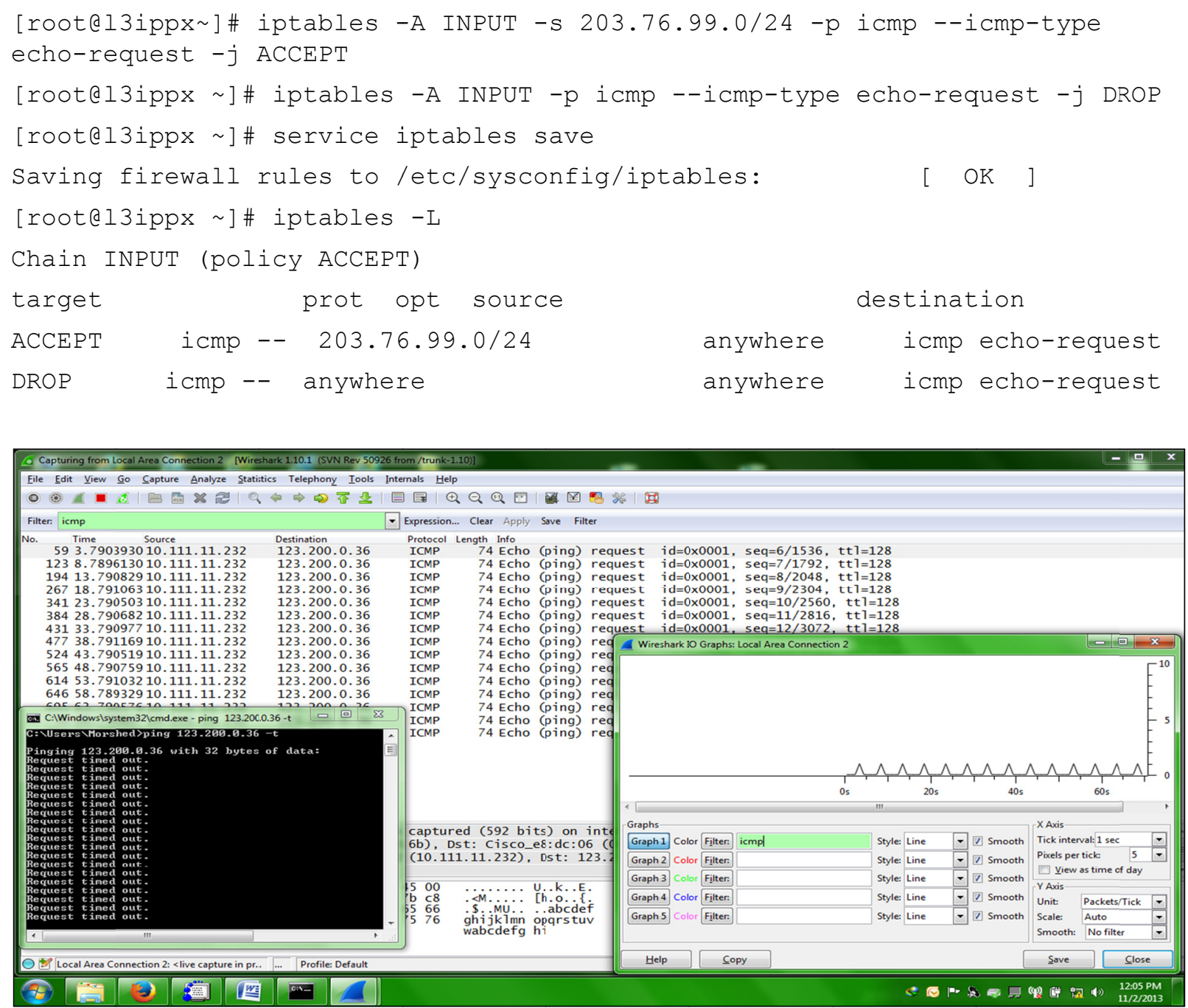

Figure 13. Wireshark view for the ping request form host 10.111.11.232 to Linux server with IP address 123.200.0.36 and and packet/tick graph at wireshark

Now the ping request sender 10.111.11.232 will get request time out as an acknowledgment message from the host 123.200.0.36 because Linux kernel will dropped the ping request packets as the kernel allow only the IP block 
203.76.99.0/24 and there will be no reply request packet to the host 10.111.11.232 from 123.200.0.36 as wireshark flow graph shows at Figure 13. Therefore the Packet/Ticks graph Figure 14 at wireshark for icmp ping request shows the discontinuity of packets as the host 123.200.0.36 did not send reply ping request packets to host 10.111.11.232

\begin{tabular}{|c|c|c|}
\hline \multicolumn{3}{|c|}{ I Local Area Connection 2 - Graph Analysis } \\
\hline Time & 10.111.11.232 & Comment \\
\hline 3.790393000 & Echo (ping) request & ICMP: Echo (ping) request id $=0 \times 0001$ seq $=6 / 1536, \mathrm{tt} \mid=128$ \\
\hline 8.789613000 & Echo (ping) request & ICMP: Echo (ping) request id $=0 \times 0001$, seq $=7 / 1792, \pi t=128$ \\
\hline 13.790829000 & Echo (ping) request & ICMP: Echo (ping) request id $=0 \times 0001$, seq $=8 / 2048, t t=128$ \\
\hline 18.791063000 & Echo (ping) request & ICMP: Echo (ping) request id $=0 \times 0001$ seq $=9 / 2304, t t \mid=128$ \\
\hline 23.790503000 & Echo (ping) request & ICMP: Echo (ping) request id $=0 \times 0001$ seq $=10 / 2560, \pi t=12 \varepsilon$ \\
\hline 28.790682000 & Echo (ping) request & ICMP: Echo (ping) request id $=0 \times 0001$, seq $=11 / 2816, \pi t=12 \varepsilon$ \\
\hline 33.790977000 & Echo (ping) request & ICMP: Echo (ping) request id $=0 \times 0001, \operatorname{seq}=12 / 3072, \pi t=12 \varepsilon$ \\
\hline 38.791169000 & Echo (ping) request & ICMP: Echo (ping) request id $=0 \times 0001, \operatorname{seq}=13 / 3328, \pi t=12 \xi$ \\
\hline
\end{tabular}

Figure 14. Wireshark flow graph for the ping request form host 10.111.11.232 to Linux server with IP address 123.200.0.36

Now the trace route command by host 10.111.11.232 shows the request time out response and failed to reach the SIP server 123.200.0.36 as given in Figure 14 due to applied rules for ICMP at Linux iptables.

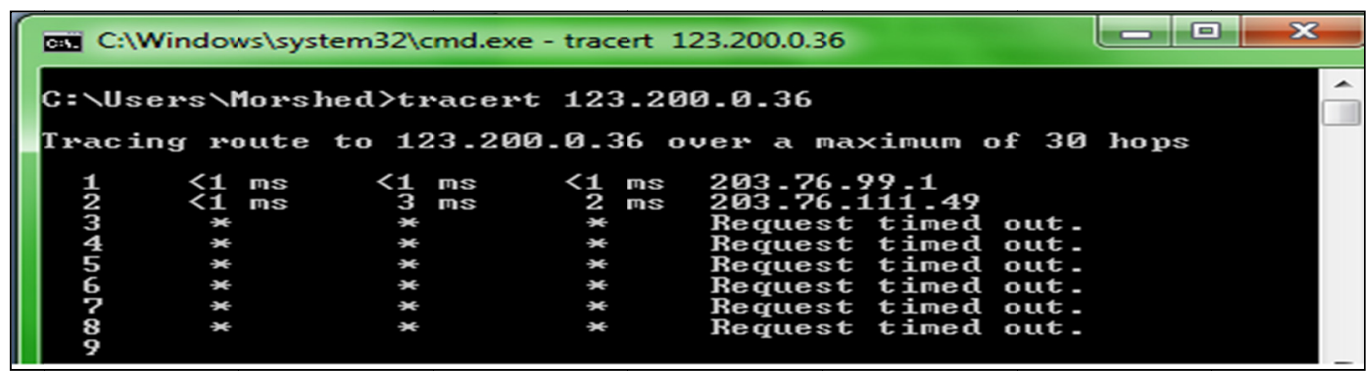

Figure 15. Trace route path form the host 10.111.11.232 to SIP server 123.200.0.36 after applying ICMP firewall

Linux iptables show the accepted and dropped packets according to apply rules as like as bellow command output in Figure 16.

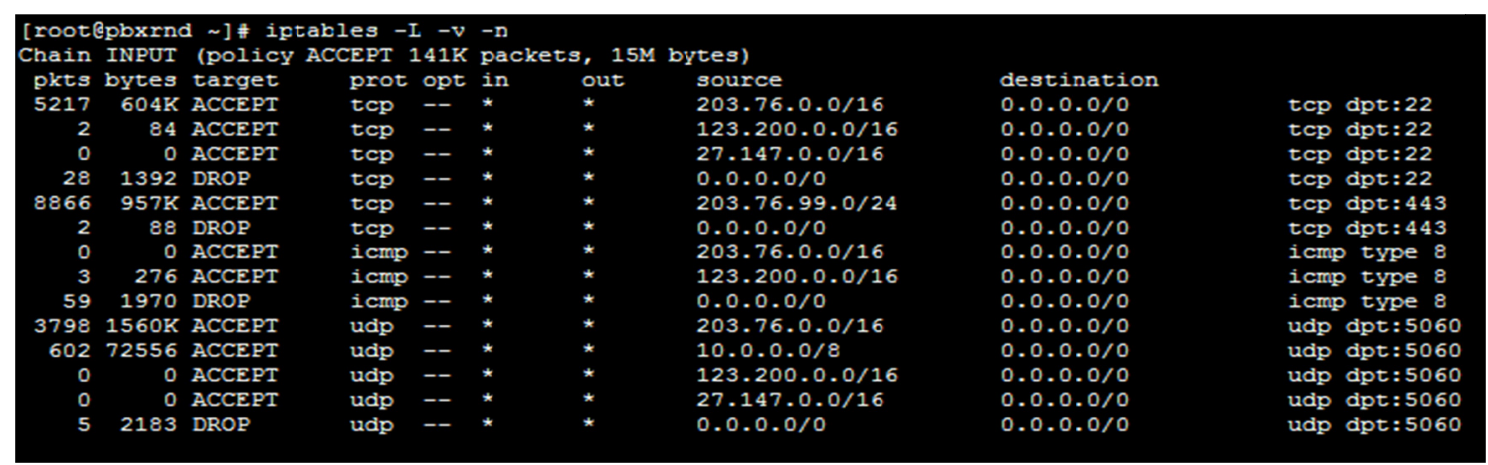

Figure 16. Accepted and dropped packets list according to rules applied at Linux firewall/iptables 
Administrator can delete existing rules at Iptables through following steps as given below. User has to give the command to see the iptables rules with the line number.

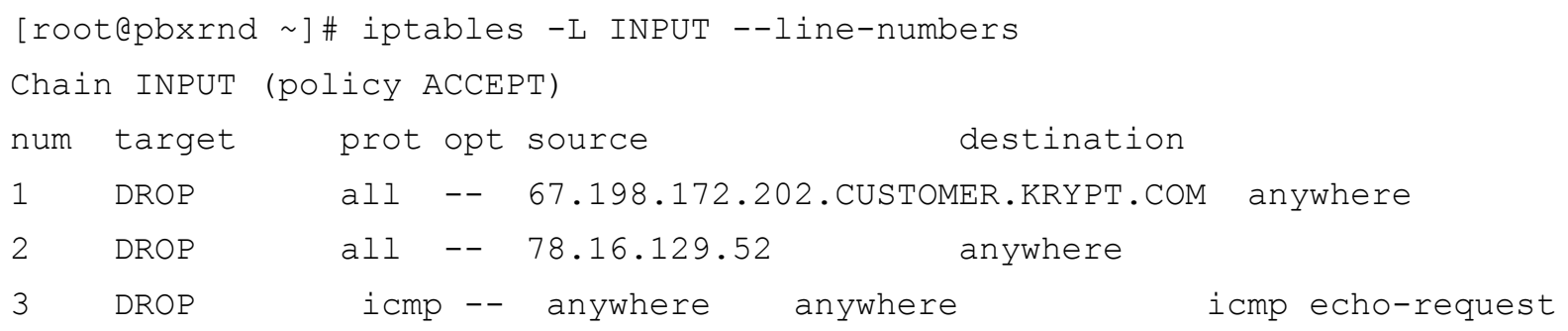

Suppose user wants to delete the rules number 2, then follow steps given bellow:

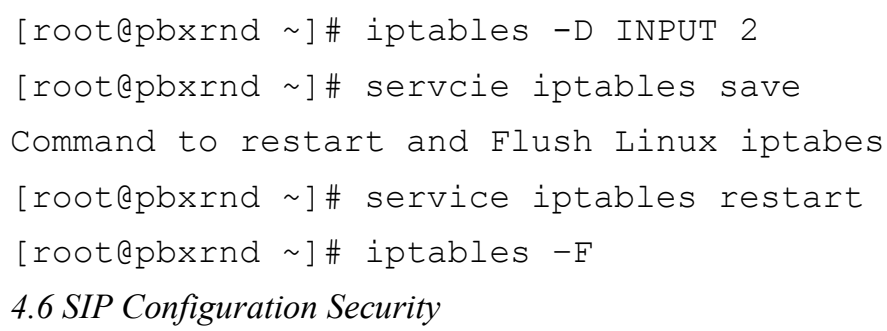

To make certain security for each static extension at sip.conf file of asterisk, administrator can set each extension parameter value safer like do not accept SIP authentication requests from all IP addresses. Utilize the "permit" and "deny" lines in sip. conf to only permit an acceptable subset of IP addresses to attain each listed extension/user. For instance parameter "permit $=10.111 .0 .0 / 255.255 .0 .0$ " is only allowed this kind of subset of IP addresses for SIP register authentication to the asterisk server others IP or IP ranges is going to be blocked. Use strong passwords for SIP entities. To defend asterisk server from password estimating attacks, always ensure that all SIP client anecdotes have a strong password at smallest 6 characters with a blend of top and smaller case notes in addition to numeric digits and at least one non-standard feature such as for example $\$, !, \#, *$, etc. For instance secret=M0r\$H3d!100 can be quite a powerful password. To ascertain the set of user anecdotes and passwords in asterisk, run the order "sip display users" at the asterisk CLI.

Always use "type=peer" and not ever "type=friend": When supplementing static SIP gazes or "SIP Trunks" in FreePBX/ Trixbox/ Elastix set the kind to "peer" and not ever set it to "friend". Utilizing "type=friend" will make asterisk server much more vulnerable because "type=friend" really determinants two objects to be conceived a SIP gaze and a SIP user. By far the poorest error that a manager could make when defining a static SIP peer would be to have both "type=friend" and "insecure=invite". In this status, a hacker could kick off calls from any isolated IP address without needing to authenticate with a password. They would only require to supposition one part of data the client name.

\subsection{Extensions Security Using Fail2ban in SIP Server}

Fail2ban operates by monitoring log files (e.g. /var/log/pwdfail, /var/log/auth.log, etc.) for selected entries and running scripts based on them. Most commonly this is used to block selected IP addresses that may belong to hosts that are trying to breach the system's security. It can ban any host IP that makes too many login attempts or performs any other unwanted action within a time frame defined by the administrator. Fail2ban is typically set up to unban a blocked host within a certain period, so as to not "lock out" any genuine connections that may have been temporarily misconfigured. However, an unban time of several minutes is usually enough to stop a network connection being flooded by malicious connections, as well as reducing the likelihood of a successful dictionary attack. Fail2ban can perform multiple actions whenever an abusive IP is detected: update iptables or PF firewall rules, TCP Wrapper's hosts.deny table, to reject an abuser's IP address; email notifications; or any user-defined action that can be carried out by a Python script.

It works by scanning log files and then taking action based on the entries in those logs. Fail2ban implemented with a configuration to be able to prevent SIP brute force attacks against our Asterisk PBXs. The following describes how to setup fail2ban to protect an Asterisk PBX from SIP brute force attempts and scans utilizing the iptables firewall. After install fail2band in SIP server, we need to create a configuration for Fail2Ban so that it can understand attacks against Asterisk. If having issues with system not banning properly when the "Registration from" section in your log file contains a quotation mark (") as in the Figure 17. 


\begin{tabular}{|c|c|c|}
\hline $\begin{array}{l}{[2014-01-2808: 17: 35]} \\
\text { matching peer found }\end{array}$ & : Registration & n from ' '14266"<sip:14266@202.4.96.201>' failed for '80.86.87.212' - No \\
\hline $\begin{array}{l}{[2014-01-2808: 17: 35] \quad[27229]:} \\
\text { matching peer found }\end{array}$ & : Registration & I from ' '14267"<sip:14267@202.4.96.201>' failed for '80.86.87.212' - No \\
\hline $\begin{array}{l}{[2014-01-2808: 17: 35] \quad[27229]:} \\
\text { matching peer found }\end{array}$ & : Registration & I from ' '14268"<sip:14268@202.4.96.201>' failed for '80.86.87.212' - No \\
\hline $\begin{array}{l}{[2014-01-2808: 17: 35] \quad[27229]:} \\
\text { matching peer found }\end{array}$ & : Registration & I from ' '14269"<sip:14269@202.4.96.201>' failed for '80.86.87.212' - No \\
\hline $\begin{array}{l}{[2014-01-2808: 17: 35] \quad[27229]:} \\
\text { atching peer found }\end{array}$ & : Registration & n from ' '1427"<sip:1427@202.4.96.201>' failed for '80.86.87.212' - No m \\
\hline $\begin{array}{l}{[2014-01-2808: 17: 36] \quad[27229]:} \\
\text { matching peer found }\end{array}$ & : Registration & n From ' '14270"<s1p:14270@202.4.96.201>' falled for '80.86.87.212' - No \\
\hline $\begin{array}{l}{[2014-01-2808: 17: 36]} \\
\text { matching peer found }\end{array}$ & : Registralion & n from ' '14271"<sip:142710202.4.96.201>' failed for '80.86.87.212' - No \\
\hline $\begin{array}{l}{[2014-01-28 \text { 08:17:36] } \quad[27229]:} \\
\text { matching peer found }\end{array}$ & : Registration & n from ' '14272"〈sip:142720202.4.96.201〉' failed for '80.86.87.212' - No \\
\hline $\begin{array}{l}\text { [2014-01-28 } 08: 17: 36] \quad[27229]: \\
\text { matching peer found }\end{array}$ & : Registration & 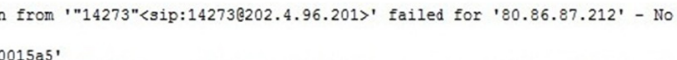 \\
\hline $\begin{array}{l}{[2014-01-2808: 17: 36]} \\
\text { matching peer found }\end{array}$ & : Registra & I from '"14274"<sip: 142740202.4 .96$. \\
\hline
\end{tabular}

Figure 17. SIP flood attacks

To prevent SIP flood by fail2ban need to create a new filter configuration for Asterisk:

touch /etc/fail2ban/filter.d/asterisk.conf

Need to add the following line will block these attempts. Next edit/etc/fail2ban/jail.conf to include the following section so that it uses the new filter. It is also recommend to turn on an iptables ban for ssh and httpd/apache if they are running on the system.

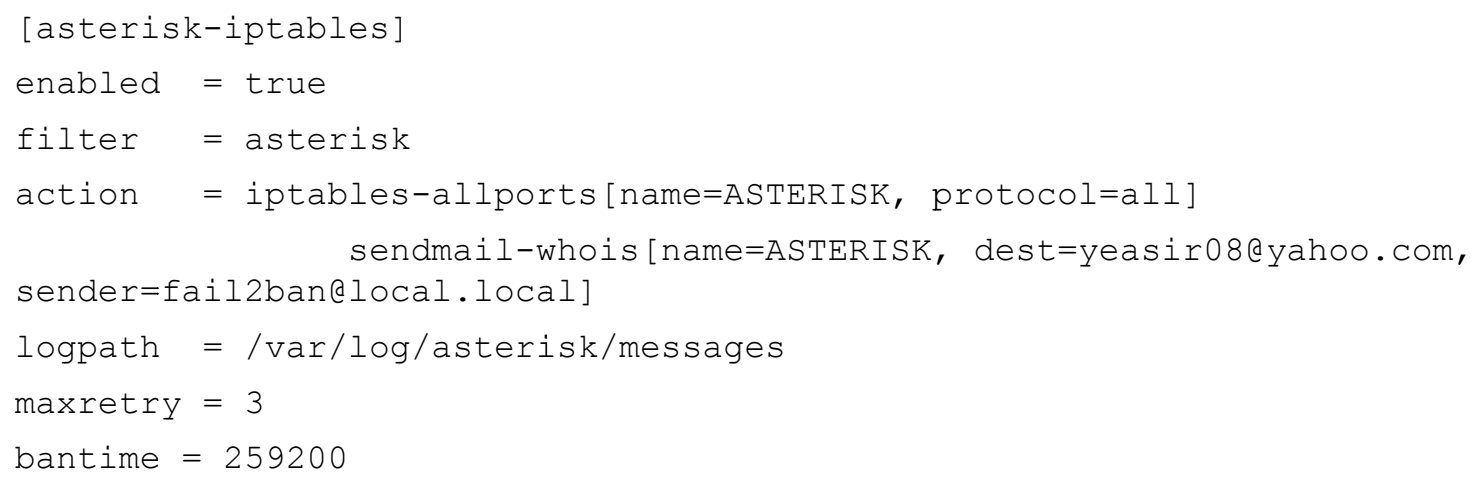

After using 3 times wrong authentication attacker IP address will automatically block in SIP server. The block IP address ate given in Figure 18.

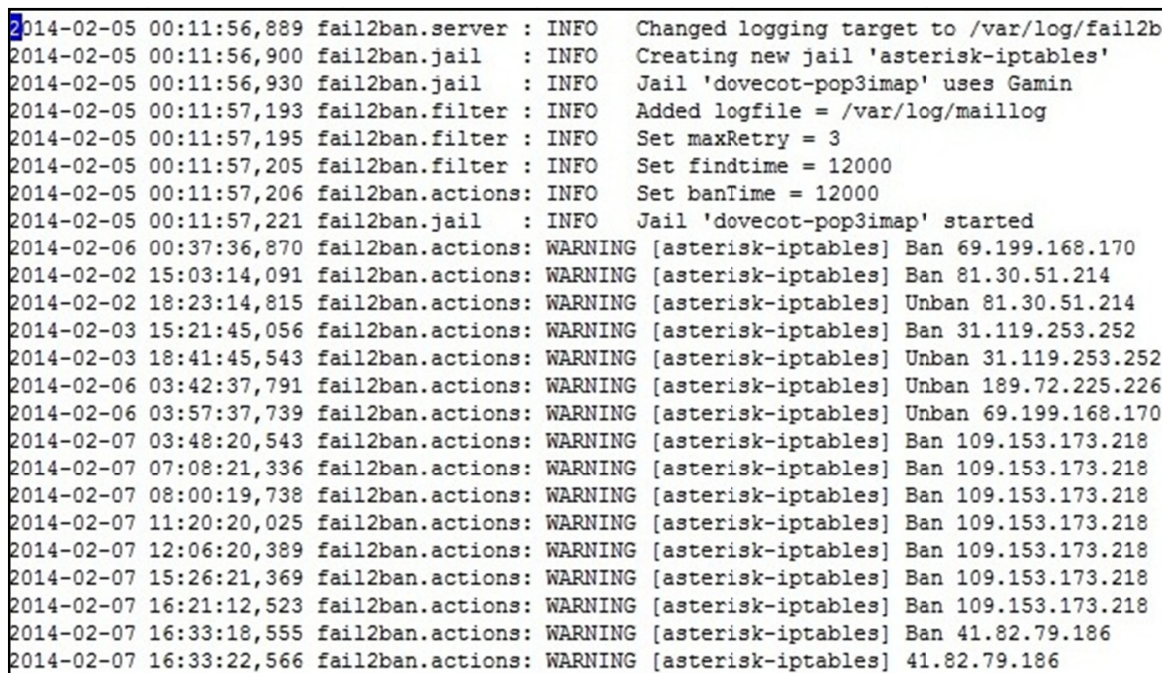

Figure 18. Attacker blocked IP address 


\section{Conclusion}

In this paper, authors focused on capability of iptables rules is explored to protect from this attack. To verify if the network traffic is legitimate or not, associate degree iptables depends on a set of rules it has which can be predefined by way of a network or system administrator. Major concentration of the paper has been on apprehending the live traffic mistreatment the network protocol analyzer Wireshark and on the premise of study scripts mistreatment iptables are developed to allow/deny the network traffic relying upon the traffic rate of any IP address of the computer inducing the packets. Authors have analyzed how Linux iptables rule work with wireshark simulation showing the difference of filtering packets before and after applying rules. Here authors ensured security for port number and some useful protocols such as SIP, SSH, ICMP, HTTPS by filtering TCP and UDP packets with allowing a particular IP addresses with subnet mask and disallowing rest of the addresses of the world to prevent unauthorized attack to the VoIP server. Linux log files which situated at /var directory is more useful to monitor the SIP server. This paper also shows how to choose strong password pattern that ensured more security to the SIP server.

\section{Acknowledgements}

The authors would like to thanks Department of Electrical and Electronic Engineering, School of Engineering and Computer Science, Independent University, Bangladesh.

\section{References}

Allawi, D., Rohiem, A. A., El-moghazy, A., \& Ghalwash, A. (2013). New Algorithm for SIP Flooding Attack Detection.

AL-Musawi, B. Q. M. (2012). MITIGATING DoS/DDoS ATTACKS USING IPTABLES. International Journal of Engineering \& Technology, 12(3).

Alqahtani, A. H., \& Iftikhar, M. (2013). TCP/IP Attacks, Defenses and Security Tools. International Journal of Science and Modern Engineering (IJISME), 1(10).

Arafat, M. Y., Ahmed, F., \& Sobhan, M. A. (2013). SIP Security in IP Telephony. International conference at Elastix World Mexico, October, 2013.

Chatterjee, K. (2013). Design and Development of a Framework to Mitigate DoS/DDoS Attacks Using IPtables Firewall. International Journal of Computer Science and Telecommunications, 4(3).

Chen, S., Xu, J., Kalbarczyk, Z., Iyer, R. K., \& Whisnant, K. (2004). Modeling and evaluating the security threats of transient errors in firewall software. Performance Evaluation, 56(1), 53-72. http://dx.doi.org/10.1016/j.peva.2003.07.013

Dantu, R., Fahmy, S., Schulzrinne, H., \& Cangussu, J. (2009). Issues and challenges in securing VoIP. Computers \& Security, 28(8), 743-753. http://dx.doi.org/10.1016/j.cose.2009.05.003

Ehlert, S., Geneiatakis, D., \& Magedanz, T. (2010). Survey of network security systems to counter SIP-based denial-of-service attacks. Computers \& Security, 29(2), 225-243. http://dx.doi.org/10.1016/j.cose. 2009.09.004

El-Moussa, F., Mudhar, P., \& Jones, A. (2010). Overview of SIP attacks and countermeasures. In Information Security and Digital Forensics (pp. 82-91). Springer Berlin Heidelberg. http://dx.doi.org/10.1007/978-3642-11530-1_10

Enhancing Network Security in Linux Environment. (2012). Technical Report, IDE1202, February 2012

Liu, L. (2010). Security Analysis of Session Initiation Protocol - A Methodology Based on Coloured Petri Nets. 1st International Cyber Resilience Conference, Australia.

Rani, D. D., Krishna, T. S., Dayanandam, G., \& Rao, T. V. (2013). TCP Syn Flood Attack Detection And Prevention. International Journal of Computer Trends and Technology (IJCTT), 4(10), 3412.

Sharma, B., \& Bajaj, K. (2011). Packet Filtering using IP Tables in Linux. International Journal of Computer Science Issues (IJCSI), 8(4).

Taluja, S., Verma, P. K., \& Dua, R. L. (2012). Network Security Using IP firewalls. International Journal of Advanced Research in Computer Science and Software Engineering, 2(8).

Voznak, M., \& Safarik, J. (2012). Dos Attacks Targeting SIP Server and Improvements of Robustness. International Journal of Mathematics and Computers in Simulation, 6(1). 
WM Eddy. (2007). RFC 4987 - TCP SYN Flooding Attacks and Common Mitigations.

\section{Copyrights}

Copyright for this article is retained by the author(s), with first publication rights granted to the journal.

This is an open-access article distributed under the terms and conditions of the Creative Commons Attribution license (http://creativecommons.org/licenses/by/3.0/). 